\title{
Probleme, Aufgaben und Ziele DER ANALYSE POPULÄRER MUSIK
}

\author{
André Doehring
}

»But analyze we must« (Walser 2003: 21).

\section{Zur Situation der wissenschaftlichen Beschäftigung mit Analyse}

Es erstaunt, wie ruhig die deutschsprachige Diskussion über die Analyse populärer Musik im Vergleich zum anglophonen Bereich (bspw. Middleton 1990; Brackett 1995; Everett 2000; Moore 2003a, 2003b; Frith 2004; Warner 2009) geblieben ist, was auf unterschiedliche Gewichtungen des Gegenstands wie auch eine divergierende Sensibilität für die daraus sich ergebenden Fragen und Probleme deuten mag. Der Blick in die maßgeblichen Nachschlagewerke der Musikwissenschaft belegt dies: Der Artikel in der MGG (Gruber 1994) widmet der Analyse vierzehn Spalten, die sich über acht Seiten erstrecken. Immerhin hat sich somit der Umfang, gewertet als Indiz für die dem Thema beigemessene Bedeutung, im Vergleich zur ersten Ausgabe (Erpf 1951) ungefähr verdreifacht. Allerdings ist er immer noch als gering zu werten, zieht man den Artikel über Analyse im New Grove (Bent/Pople 2001) hinzu, der sich über 63 zweispaltige Seiten erstreckt, in denen sowohl eine historische als auch eine problemzentrierte Perspektive auf Analyse eingenommen werden. Diese Form und das Ausmaß der Auseinandersetzung als Ergebnis der New bzw. der Critical Musicology zu deuten, dürfte sicherlich nicht falsch sein. Im Übrigen erscheint es ebenso bezeichnend wie eigentlich - da erwartbar - unnötig zu erwähnen, dass eine Auseinandersetzung mit der Analyse populärer Musik, ja selbst ein Verweis darauf im deutschsprachigen Artikel nicht entdeckt werden kann. Der New Grove schneidet die Thematik im Analyse-Artikel in einem knappen Absatz an (ebd.: 564), im dortigen Artikel zur populären Musik finden wir, neben kaum 
diskutierten Formanalysen oder Harmoniepattern (Middleton 2001: 142ff.), ebenfalls einen Absatz (ebd.: 151) bzw. eine halbe Seite (Manuel 2001: 159) über die Methoden und Probleme der Analyse populärer Musik. Angesichts des Ausbleibens von Analyse betreffenden Erörterungen im Artikel zur populären Musik in der MGG (Wicke 1997) - auch dieser ist kürzer als das englischsprachige Pendant - kann sich der Zusatz »immerhin« kaum verkniffen werden.

Im Feld der deutschsprachigen Popularmusikforschung, die zwar als interdisziplinär angelegtes Unternehmen nicht allein, aber doch überwiegend der Musikwissenschaft entstammt, ${ }^{1}$ ist man ein wenig sensibilisierter für die besondere Herausforderung der Analyse nicht-notierter populärer Musik. Denn der im Vergleich zur Analyse westlicher Kunstmusik fehlende Notentext sowie die vielfältigen Möglichkeiten der Verwendung einer Musik in unterschiedlichsten Kontexten haben an der Nützlichkeit der Methodik von Analyse zweifeln lassen, die bisher vorrangig dazu diente, »in der Musik« nicht nur den Kunst- und Werkcharakter der untersuchten Gegenstände, sondern zugleich die eigene Disziplin als Kunstwissenschaft zu legitimieren. Aufgeworfene Fragen betrafen sowohl Mängel der Methodik, der Theorie als auch die Grenzen des untersuchten Gegenstands und somit der Möglichkeiten des disziplinären Zugriffs (vgl. Helms 2002; Wicke 2002). Zudem scheint das bisher analysierte Repertoire kaum geeignet, den umfassenden Terminus »populäre Musik« in seiner Breite abzubilden, denn noch immer zeichnen sich populärmusikalische Analysen durch einen engen zeitlichen und stilistischen Rahmen aus, was in erster Linie an den Auswählenden ${ }^{2}$

1 Dies ist sicher eine Folge der besonderen Entwicklung der deutschen systematischen Musikwissenschaft seit etwa dem Ende der 1960er Jahre, in der sich eine jüngere Generation von Wissenschaftlern mit einem erweiterten wissenschaftlichen Instrumentarium neuen und auch populärmusikalischen Gegenständen zugewandt hatte. Bereits im ersten Band der Beiträge zur Popularmusikforschung war es Ekkehard Jost (1986: 36), der, neben dort vertretenen Autoren wie Hermann Rauhe, Günter Kleinen oder Helmut Rösing, speziell auf den Aspekt der Analyse populärer Musik als eine Lösung für die »Probleme der Popularmusikforschung « verweist.

2 Dasjenige Repertoire wird behandelt, das man kennt und - in aller Regel wertschätzt. Sehr wahrscheinlich ist diese Fokussierung in Zusammenhang mit der beginnenden Institutionalisierung der deutschsprachigen Popularmusikforschung in den 1980er Jahren zu sehen. Hier bemühte sich eine (auch) mit Rockmusik sozialisierte Generation von Musikwissenschaftlern, das neue Forschungsgebiet methodisch zu etablieren, indem sie an den gewählten Stücken der Beatles oder des Progressive Rock das legitimierte und legitimierende Handwerkszeug der Analyse anwandten (ich danke Alfred Smudits für diesen Hinweis). Künftige Generationen von Musikwissenschaftlern werden demnach »ihre und somit eventuell andere Musik untersuchen. Allerdings muss man skeptisch sein: Regelmäßige Befragungen der Studienanfänger in meinen Semi- 
selber sowie den verwendeten theoretischen Voraussetzungen und Methoden (dazu später mehr) der Analyse liegen dürfte. Viele Bereiche sind fast nicht präsent: etwa der stets nur mit spitzen Fingern angefasste Pop, der die Charts dominiert, oder die gesamte Palette der elektronischen Tanzmusik, die sowohl als zu neu (»Ist doch bereits morgen wieder vergessen!«), zu redundant (»Lohnt sich das denn >musikalisch überhaupt für eine Analyse?«) oder gar überhaupt nicht behandelbar mit den Werkzeugen der Musikwissenschaft gilt. ${ }^{3}$ Außerdem kaum zur Analyse gelangen beispielsweise Live-Darbietungen, womit das gesamte Konzert- bzw. Clubgeschehen und somit auch der Bereich improvisierter populärer Musik sowie große Anteile des Amateur-Musikbereichs aus dem Fokus oder vielmehr: erst gar nicht in den Fokus der Wissenschaft geraten.

Denn nach wie vor gilt der im Tonstudio aufgenommene Tonträger als Ausgangs- und Bezugspunkt der Analyse populärer Musik - der in aller Regel als Produkt (i.e. Ware) einer musikindustriellen Handlung durch Verknüpfung verschiedener Produktionsbereiche den Weg zum Analysierenden ${ }^{4}$ gefunden hat, was in der Analyse oft verschwiegen oder nur unzureichend reflektiert wird. Und somit lockt den Musikologen stets die Versuchung bzw. es dräut die Gefahr, das hier vorliegende Stück Musik ähnlich dem in der Analyse abendländischer Kunstmusik vorliegenden Notentext als geschlossene Einheit, gar als >Werk < zu überhöhen, das vom >Künstler - Subjekt oder -Team exakt so und nicht anders auf Band resp. Festplatte aufgenommen wurde. ${ }^{5}$ Ähnlich sieht Peter Wicke (2003: 117) die im Analyseprozess entstehende Notation von populärer Musik als »überaus problematisch « an, weil sie als ein schriftliches Medium Klingendes - unzureichend - festzuhalten

naren legen genau dieselben Muster der Geringschätzung von Stilbereichen an den Tag, die in der Wissenschaft gepflegt werden, nämlich nachdrückliche Abneigungen von afroamerikanisch geprägten Stilbereichen (HipHop, R'n'B) oder elektronischer Tanzmusik. Offensichtlich reproduzieren sich hier Geschmacksmuster einer gebildeten bzw. sich in der Ausbildung befindenden Mittelschicht, die, ähnlich wie Bethany Bryson (1996) dies einst für den Heavy Metal oder die Country Music feststellte, eine Distinktion nach unten vollführt, d.h. sich via Geschmack resp. »geschmäcklerisch « von der mit »niederen« sozialen Schichten assoziierten Musik distanziert.

3 Darunter fallen beispielsweise die Fragen, wie Klangerzeugung und -bearbeitung dieser Musik erfasst, dargestellt und somit einer Interpretation zugänglich gemacht werden können. Erschöpfende Antworten können hier kaum gegeben werden, es deutet sich aber ein weiterer Diskussions- und Forschungsbedarf an.

4 Die im Artikel durchweg gebrauchte männliche Form ist weniger dem auch in der Musikwissenschaft herrschenden grotesk asymmetrischen Geschlechterverhältnis geschuldet als der besseren Lesbarkeit.

5 Vgl. bspw. die Analyse von »A Day In The Life« (1967) der Beatles (Geuen/ Hiemke 2001). 
sucht. Für eine Aufführungspraxis, in der Notation als Ausgangspunkt musikalischer Produktion dient, mag dieses Verfahren sinnvoll erscheinen. In einer Musikkultur allerdings, die weitgehend schriftlos - selbst nach der Notation durch den Musikwissenschaftler - musiziert, erscheint diese Notation als Endpunkt des nicht-notierten Musikprozesses doppelt unangemessen. Auch Wicke warnt vor der benannten musikwissenschaftlichen déformation proféssionelle: Ist Klang erst einmal in westlicher Notation allein geronnen, droht selbst dem widerständigsten Musikologen die Gefahr, der »Diktatur des tonalen funktional-harmonischen Systems « (ebd.) zu erliegen, sprich: er drückt sich in den erlernten und mithilfe der Noten ja belegbaren Termini aus, ohne dies in angemessener Weise zu reflektieren. So bleibt zunächst festzuhalten: Gegenstand der Analyse ist eine klangliche Struktur, deren Notation im Prozess der Analyse populärer Musik lediglich ein Hilfsinstrument, ein Mittel und kein (Selbst-)Zweck ist.

In den letzten Jahren konnte man zu dem Eindruck gelangen, dass die wissenschaftliche Beschäftigung mit der Analyse populärer Musik in quantitativer Hinsicht trotz dieser vereinzelt geäußerten Bedenken eindrucksvoll gestiegen ist. $^{6}$ In neueren Arbeiten (Elflein 2010, Rappe 2010) immerhin avancieren bisher vernachlässigte Stilbereiche zum Analysegegenstand. Hier findet man ausgreifende kulturelle Kontextualisierungen des Klingenden, sensibilisierte Überlegungen bezüglich methodischer und technologischer Aspekte der Erfassung von Sound oder stimmlicher Darbietung sowie deren Darstellung in Transkriptionen. Und doch bleibt noch immer eine für den deutschsprachigen Raum insgesamt problematische Literaturlage zu beklagen, in der nicht zuletzt umfassende Hand- oder Lehrbücher fehlen und somit kaum exemplarische Analysen vorhanden sind, die vorbehaltlos Studierenden empfohlen werden könnten, die sich in analytischen Seminaren und Arbeiten mit der Analyse populärer Musik beschäftigen.

\section{Die Öffnung des Musikbegriffs}

Vor diesem Hintergrund möchte ich im Folgenden darlegen, dass eines der zentralen, weil grundlegenden Probleme der Analyse populärer Musik (immer noch) der Musikbegriff ist, der den meisten Analysen zugrunde liegt. Der Musikwissenschaftler Andreas Moraitis (1994: 166) verdeutlicht diese

6 Jüngstes Indiz für diese Hinwendung zur Analyse populärer Musik ist die ASPMTagung »Black Box Pop. Analysen populärer Musik« (2010), die die bisher höchste Zahl von Teilnehmern und Vortragenden in der Geschichte des ASPM verzeichnete. 
Auffassung schlechterdings, indem er Analyse und Hermeneutik voneinander abgrenzt: Analyse umfasse alle Aktivitäten, die sich »mit der rein musikalischen Faktur eines Werkes « beschäftigen, Hermeneutik dagegen behandele die »Deutung der außermusikalischen Referenzen«. Der Gegenstand einer Analyse wäre demnach als objektive, autonome Größe bestimm- und somit mit dem Instrumentarium der Analyse analysierbar, alles Äußere wäre in diesem ersten Schritt der Beschäftigung mit »der Musik allein« irrelevant, da erst in der Interpretation »externe « Umstände eine Bewandtnis erhielten. ${ }^{7}$

Dabei verhält es sich doch in Wirklichkeit so, dass Musik als diskursiver Begriff der nicht verbalisierbaren ästhetischen Erfahrung das darstellt, was eine diskursive Gemeinschaft darunter verstehen möchte bzw. soll. Im von Wicke (2002, 2003, 2004) vorgestellten zweistufigen Musikerfahrungsprozess ist der Musikbegriff deshalb von der klanglichen Struktur abgekoppelt. Der Begriff, den sich Menschen, Gruppen oder Berufszweige wie die Musikwissenschaft von Musik in diesem Sinne machen, stellt daher gerade nicht eine autonome Größe, sondern eine sozial verfestigte Art des Sprechens über Klang und Klangerfahrungen dar. ${ }^{8}$ Da die Klangerfahrung erst in Begriffen zu »Musik« wird - Wolfgang Horn (1996: 16) spricht poetisch von einer »Mitgift der Existenzweise von Musik« -, muss diese Versprachlichung eine Untersuchungsebene der Musikanalyse sein. Denn die Art des Sprechens bzw. Schreibens über Musik ist keinesfalls bloß subjektiv, sondern sie ordnet sich einem Regime des Sprechens und des - gerade in Expertenkreisen wie der musikologischen Community natürlich richtigen - Denkens über Musik unter bzw. sie kann ihm erst gar nicht entkommen. Die Nichtbegrifflichkeit von Musik macht also jede Äußerung darüber bereits zu einer diskursiven Äußerung, d.h. sie ist ebenso Teil und Ergebnis eines Diskurses, wie sie, je nach Sprecherposition, weitere Aussagen über Musik formen wird. Auch diejenige Analyse, die vermeintlich »rein musikimmanent« sich mit »der Musik« be-

7 Diese Haltung lässt sich etwa bei Allan F. Moore wiederfinden. Er unterzieht jede Analyse einem »so what?<-test, that is, how well it [analytical informed work] moves from analysis to useful, and usable, interpretation « (Moore 2009: 412). Wie im Weiteren dargelegt wird, finden wir hier zwar die geforderte Überprüfung der eigenen Position und der angestrebten Zwecke der Analyse, aber die Annahme, dass eine Kluft zwischen Analyse und Interpretation zu überspringen wäre, ist falsch: Von Anfang an sind wir, erst als Beobachter, Hörer oder Teilnehmer, dann als für die Analyse Auswählender und Interpretierender, mit Musik im Wickeschen Sinne konfrontiert. Wir können uns weder unseres Blickes auf noch des Diskurses über die untersuchte Musik in der für Moore vorrangigen ersten Analyse entziehen.

8 Christopher Small (1998) hat diesen aktiven Musikprozess, der erst durch das Handeln von Menschen ermöglicht wird, als »musicking « bezeichnet. 
schäftigt, indem sie bspw. harmonische Progressionsmodelle absolutiert, führt derart ein diskursives Regime fort. Ähnlich führt auch die verbreitete Vorstellung von Musik als Text in die Irre, da sie dazu verleitet, Bedeutungen dem »Text«bzw. der Musik zu entnehmen, wo doch diese im Umgang, d.h. in der Benennung als Text erst entstehen.

Eine Aufgabe von Musikanalyse müsste es daher sein herauszustellen, wie das jeweilige Klanggebilde als Musik in unterschiedlichen Kontexten erfahren werden kann. Tia DeNora (2000: 31) kritisiert das analytische Verfahren in ihrem Plädoyer für eine »sociology of musical affect « dafür, dass es nicht berücksichtige, welche Bedeutungen Musik als kulturelles Vehikel Wicke (2004: 116) benutzt das Bild eines »Generators« - für verschiedene Menschen in unterschiedlichen Situationen besitzen kann. Dietrich Helms (2002: 102) fordert daher zugespitzt, dass Musikanalyse eigentlich eine Analyse der Rezeptionskontexte sein müsse, d.h. »der Prozesse, welche einem Stück seine Bedeutungen zuweisen«. Somit wäre das Bild des solitären, über die Noten gebeugten Musikwissenschaftlers endgültig über Bord geworfen, da nun eine empirisch gestützte, qualitativ ausgerichtete Erfassung von Aneignungen und Bedeutungen von populärer Musik wohl angeraten scheint. Hat Analyse als Instrument der Erkenntnissuche also ausgedient?

Zunächst muss darauf hingewiesen werden, dass auch die musikwissenschaftliche Analyse einen Fall der Rezeption darstellt. Insofern kann eine wohl reflektierte Analyse - zu diesem Punkt ausführlicher unten - bereits eine mögliche Form der Rezeption analytisch und argumentativ erfassen. Zweitens ist darauf zu beharren, dass der Rezeptionskontext von Musik zwar imstande ist, neue Bedeutungen zu erzeugen, er dies aber in keinesfalls zufälliger Verknüpfung von musikalischem Material und Bedeutung tut. Deshalb betont auch Helms (ebd.), dass der Kontext stets "gleichzeitig auf struktureller bzw. materialer, auf individuell-psychischer und auf sozialer Ebene « verortet werden muss.

Gerade weil die klangliche Basis wichtig ist für die jeweilige Rezeption dieser Musik, ohne in irgendeiner Weise determinierend zu wirken, verlangt ein derart erweiterter Musikbegriff vom Wissenschaftler des Weiteren nicht allein das Bewusstsein der sozialen Beschränktheit der eigenen Begrifflichkeit, sondern er muss sich über die benutzten und an die Musik herangetragenen Begriffe ständig Rechenschaft ablegen, da er das klangliche Objekt der Analyse durch die Art des Sprechens diskursiv rahmt - wie auch alle anderen, die über dieses Objekt sprechen. ${ }^{9}$

9 Vgl. auch die Ausführungen von Cook (2001). 
»The act of description [...] co-produces itself and the meaning of its object. With regard to music, then, the matter of its social significance is not pregiven, but is rather the result of how that music is apprehended within specific circumstances « (DeNora 2000: 23, Hervorhebung im Original).

Wicke formuliert daher als Aufgabe an die Popularmusikforschung, »in den kulturellen Zusammenhängen um die analysierten Musikformen und den darin jeweils dominanten Diskursen den Codes nachzugehen, die das Klanggeschehen strukturieren « (Wicke 2003: 119). Dies stellt freilich hohe Anforderungen an den Analysierenden: Entweder verfügt er über kontextuelles Wissen aufgrund seiner sozialen Verortung innerhalb der Musikkultur, deren Musik er analysiert, oder er muss es, bspw. auf ethnografischem Wege, herstellen.

Von Adorno (2003a: 253) ist das schöne Bonmot überliefert: »Sprache interpretieren heißt: Sprache verstehen; Musik interpretieren: Musik machen. "Wer demnach als musikalisch Handelnder agiert, weiß um die kontextuellen, sozialen, politischen und klanglichen Bedeutungen, ihre Voraussetzungen wie auch Folgen.

»Nur in der mimetischen Praxis [des Musizierens], die freilich zur stummen Imagination verinnerlicht sein mag nach Art des stummen Lesens, erschließt sich Musik; niemals einer Betrachtung, die sie unabhängig von ihrem Vollzug deutet« (ebd.).

Natürlich ging es Adorno als ausgebildetem Instrumentalisten und Komponisten um die Erfahrung der Aufführung abendländischer Kunst- und nicht Popularmusik, doch nimmt man diese Aufforderung nach einer von dem Vollzug musikalischer Praxis abhängigen Interpretation ernst, bedeutet es, die Kontexte der musikalischen Praxen - und hier ist ein erweiterter Begriff der musikalischen Praxis im Sinne Kurt Blaukopfs (1984: 20f.) anzulegen, der selbstverständlich über das Musizieren hinausgeht - an die Analyse heranzutragen. ${ }^{10}$

10 Dagegen erklärt - kaum überzeugend - Moraitis (1994: 296) die Stilkompetenz des Analysierenden zur Voraussetzung für ein »adäquates Musikverstehen «, worunter er die Aneignung der musikalischen als auch »musikalisch-sprachlichen « Tradition vergangener Epochen versteht. Selbstverständlich gebe es dabei Schwierigkeiten »praktischer Natur (die allerdings, richtig gewendet, theoretischer kaum sein könnten): Der Historiker habe es, etwa im Vergleich zum Musikethnologen, einfacher, Musik adäquat, also »richtig « zu verstehen, da er Stilgesetze voraussetzen könne. Der Ethnologe hingegen müsse sich vor Ort erst Wissen über die Musik aneignen. Moraitis verweist als Beispiel auf einen Artikel von Gerhard Kubik (1973) über afrikanische Musikkulturen und entblößt sich weiter: "Wir haben allerdings den Eindruck, daß Kubik die Rolle des Musikers, der vielfach unbewußt agiert, ohne über die Möglichkeit theoretischer Reflexion zu verfügen, ein wenig überbewertet« (Moraitis 1994: 296). 
Die oftmals (etwa bei Helms 2002 oder Wicke 2003) anzutreffende Warnung, dass musikwissenschaftliche Analyse ein Spezialistendiskurs sei, der die Musik ihren Kontexten entreißt und sie somit ihrer seigentlichen< Bedeutung bei den swahren Experten der jeweiligen Subkultur entledige, mag man einerseits, gerade angesichts eines eigentümlich musikologischen Jargons (etwa bei Geuen/Hiemke 2001), für zutreffend halten. Andererseits aber muss ihr entgegnet werden, dass diese Selbstmarginalisierung der Musikwissenschaft eine Priorisierung der Rezipienten (wer auch immer das sein mag?) impliziert und zur Folge hätte, dass Analyse tatsächlich zugunsten einer empirisch geleiteten Befragung ebendieser idealisierten »ExpertenHörer« ad acta gelegt werden müsste. Diese Perspektive imaginiert folglich einen gültigen Ort, eine erklärte Subjektposition und eine »wahre« Bedeutung. So wäre bspw. elektronische Tanzmusik ausschließlich vom Tänzer auf dem Dancefloor »wirklich« zu verstehen, allerdings nur dann, wenn er oder sie sich als mit allen Wassern gewaschene Mitglieder der Subkultur der Clubgänger auszuzeichnen wissen.

Jedoch gilt: einen Generalschlüssel des Musikverstehens gibt es nicht. Die musikwissenschaftliche Analyse, zugleich selbst eine mögliche Form der Rezeption, hat sich aufgrund der Sensibilisierung für andere Kontexte von Musik ihrer begrenzten Reichweite bewusst zu sein und darf sie nicht verleugnen. Sie ist und bleibt ein Spezialistendiskurs, der durch Kontextualisierung und Diskursivierung neue Erkenntniswege offen legt. Für die Analyse übrigens nicht nur - populärer Musik bedeutet dies, dass sie einen grundlegenden Paradigmenwechsel entwickeln muss. Nicht »Musik« muss untersucht werden, sondern die klangliche Ebene und die sich auf dieser Basis entfaltenden Begriffe, welche um die Deutungsansprüche über diese Musik ringen (vgl. auch Wicke 2002: 69). Die Aufgabe der Kontextualisierung verlangt, sich Wissen um die kulturellen Zusammenhänge anzueignen und es an die Analyse heranzutragen, die Forderung der Diskursivierung von Analyse betrifft die kritische Sichtung nach Machtpositionen des Sprechens über die untersuchte Musik. Beides setzt aber voraus, dass die Klangerfahrung durch den Analysierenden und ihre spezifische Art der Verbalisierung Teil eben dieses Perspektivwechsels sein müssen, wie im Weiteren ausgeführt wird. ${ }^{11}$

11 Ausgerechnet Carl Dahlhaus war sich sicher: "Man versteht Musik genauer, wenn man die Mühe nicht scheut, sich die Struktur der Sprache, in der über sie geredet wird, bewußt zu machen « (Dahlhaus 1973: 46). Dahlhaus bezieht diese Aussage auf August Halms Analyse von Beethovens d-Moll-Sonate op. 31,2, die als »Denken über Musik « ein Teil der »Sache selbst « geworden sei: »Es ist die Wirkungsgeschichte - nicht eine dem historischen Wandel enthobene ;Überwelt < - , in welcher der Sinn, der >objektivierte Geist < musikalischer Werke aufbewahrt ist « (ebd.). Letztlich sitzt Dahlhaus damit zwar auf dem richtigen Ross, 


\section{Exkurs: Vom (anfänglichen) Versagen der Analyse}

In einer Analyse von Musik der Band Maxïmo Park (Doehring 2006) ging ich der Fragestellung nach, warum ausgerechnet in dieser Band ein Wiedergänger von New Wave-Bands wie The Jam und anderen erkannt wurde und sie somit als Aushängeschild der sogenannten New Wave of New Wave galten. Im Ergebnis konnte jedoch mithilfe der gewählten Methodik der Analyse diese Frage nicht beantwortet werden. Es gab Anlass zur Vermutung, dass »außermusikalische« Faktoren wie Verpackung, Bewerbung und Lancierung - in Hermann Rauhes (1974: 18) Terminologie: die Quartärkomponenten dieser Musik eine Rolle spielten, da eben »die Musik« keine manifesten Bezüge analytisch aufzuweisen hatte. Erst durch eine ausführliche Beschäftigung (Doehring 2011) mit den medialen Prozessen und den zentralen Medienakteuren, den Musikredakteuren der für die Berichterstattung maßgeblichen deutschen Popmusikzeitschriften, die zur Bekanntheit dieser Band beigetragen hatten, kann nun ein multiperspektivischer Blick auf das Entstehen dieser Zuschreibungen an die Musik eingenommen werden.

Die in der musikalischen Analyse erkannte klangliche Struktur der Musik Maxïmo Parks weist Brüche und bewusst gesetzte Formungen auf, die den Musikjournalisten die Einordnung nach professionell erworbenen Mustern der Selektion neuer Popmusik erlaubte. ${ }^{12}$ Diese Muster der musikalischen Wahrnehmung ähneln einander nicht nur aufgrund ihres gemeinsamen institutionellen Kontextes der Anwendung - nämlich in den Redaktionen der Musikzeitschriften -, sondern auch, weil die Musikredakteure fast gleich alt und alle männlich sind und zudem über einen ähnlichen Sozialisations-, Bildung- wie Ausbildungs- und - viel wichtiger - musikalischen Habitus verfügen (vgl. ebd.: 169ff. u. 185ff.). Die spezielle Musikwerdung der klanglichen Struktur der Songs dieser Band wurde somit zunächst auf der subjektiven, dann der institutionellen wie schließlich der veröffentlichten und letztlich öffentlichen Ebene durch diese Faktoren bestimmt. Gleichzeitig haben in diesen Prozess der musikalischen Bedeutungskonstitution ökonomisch moti-

ohne allerdings tatsächlich loszureiten. Denn immer noch ist ein Sinn dem Werk zu entnehmen, das hier um seine »Wirkungsgeschichte (zudem in einer ziemlich eindimensionalen Kommunikationsvorstellung) erweitert wird.

12 Sie beschreiben die Musik der Single »Apply Some Pressure oder »eingängig «, eine Musik, die »es schaffen konnte« (vgl. Doehring 2011: 268). Die Analyse dieser Musik (Doehring 2006) belegt auf formaler, harmonischer, melodischer wie rhythmischer Ebene den erklärten Willen der Band, einen Song zu schreiben, der aus vier Refrains bestehe, um somit im Radio für einen nachhaltigen Eindruck zu sorgen. 
vierte Diskursinstanzen eingegriffen: Die Promoter und die Plattenfirma Warp haben in diesem Fall eine umfassend angelegte und kooperative (d.h. sowohl von Band- als auch Medienseite wohlwollend »begleitete«) Kampagne gestartet, die die professionellen Wahrnehmungsinstrumente der Musikjournalisten wecken, das Interesse aufrechterhalten und sie vor allem mit Begrifflichkeiten des erwünschten Sprechens über diese Musik versorgen konnte. Zusätzlich profitierte die Kampagne von einer dem musikjournalistischen Feld eigenen Dynamik der Konkurrenz: In der von den Musikjournalisten geäußerten Wahrnehmung der damaligen Situation seien »alle« von dieser Band begeistert gewesen, man »musste etwas machen« und hat zugleich die von der Plattenfirma angebotene - kostenlose - Reise zu PromotionKonzerten der Band im Ausland gerne wahrgenommen (vgl. ebd.: 266ff.).

Zusammenfassend haben habituelle, professionelle, strukturelle und ökonomische Faktoren derart auf die Musikjournalisten eingewirkt, dass sie den Klängen von Maxïmo Park diese Musikwerdung zukommen ließen - die wiederum Ausgangspunkt meiner eigenen Analyse war und damit notwendig als »musikimmanente « Analyse scheitern musste. Denn zum Zeitpunkt meiner analytischen Arbeit bin ich mir weder der benutzten Begriffe und ihrer diskursiven Prägung noch - und hier komme ich zum zweiten Punkt der Problematik der Analyse - meines Blickes, meiner speziellen Perspektive und Interessen an der Musik von Maxïmo Park bewusst gewesen.

\section{Das Forschersubjekt}

Hans Heinrich Eggebrecht (2004) hat in einem späten, zugleich hellsichtigen wie esoterisch-versponnenen Artikel über das Verstehen durch Analyse vom Analysierenden das unbedingte Bewusstwerden des subjektiven Zugangs zum »Werk« eingefordert. Eggebrecht geht, ähnlich wie Wicke, von einem zweistufigen musikalischen Verstehensprozess aus. Die erste Stufe der klanglichen Erfahrung bezeichnet er als »ästhetisches Verstehen«, die begrifflich geprägte kognitive Verarbeitung dieser Erfahrung dann als »erkennendes Verstehen«. Letzteres ist als ein Versuch der Übersetzung der vorausgehenden sinnlichen Erfahrung von Klang zu verstehen, der in der Totale, d.h. als Abbildung sämtlicher sinnlicher Empfindungen des intuitiven ästhetischen Verstehens, misslingen müsse.

Da Eggebrecht als Gegenstand der Analyse einen »in der Regel zum Klingen bestimmten Notentext « (ebd.: 18; Herv. A.D.) definiert, basiert jede Analyse als besondere Form des erkennenden Verstehens auf der subjektiven sinnlichen Erfahrung dieses Klanges. Die in der Analyse zu leistende 
Aufgabe definiert er daher als eine kenntlich zu machende Reflexion der persönlichen Zugangsweise zur Musik, der für die Analyse benutzten Begriffe sowie der daraus beeinflussten Ergebnisse der Analyse. Eine Analyse sei als gelungen zu werten, wenn sie als ein Stück »dokumentierter Rezeption « (ebd.: 20) in dieser individuellen Deutung einen Aspekt des »Werkes « anderen verständlich und nachvollziehbar darstellen kann, oder in Eggebrechts Worten: wenn das >Werk < in der Analyse »getroffen ist, und das heißt, ob auch nur als Widerschein - ein Strahl aus seiner Mitte heraus zum Leuchten kommt« (ebd.). So luzid Eggebrecht den Anteil des Forschersubjekts am Prozess der Analyse herausstellt, so fragwürdig erscheint doch sein Bezugspunkt der Analyse im »Werk«, das nach oben dargelegtem Musikbegriff durchaus als ein zu hinterfragender Gegenstand der Analyse, aber kaum als ihr objektiver Maßstab eine Rolle spielen kann.

Auch Moore (2007: xx) stellt die »necessity of self-interrogative writing « in der Analyse heraus. Diese Hinterfragung der Subjektposition des Analysierenden, ein »relatively new topic in musicology« (ebd.: ixx), eröffnet bzw. erinnert an eine ethische Dimension wissenschaftlicher Arbeit, indem sie eben nicht mehr eine, nämlich die eigene resp. die dem »Werk « »angemessene« Hörerposition verabsolutiert, sondern sich auch anderen Hörweisen öffnet und sie für die eigene Arbeit in Betracht zieht.

Genau genommen bedeutet die Einforderung von Reflexivität bezüglich der unhintergehbaren Subjektivität des analytischen Zugriffs auf Musik, dass der Musikwissenschaftler sich nun die Fragen tatsächlich stellen bzw. gefallen lassen muss, nicht nur wie, sondern auch für wen und somit wofür er es denn tut. Diether de la Motte bspw. antwortet auf die Frage von Schüler (1996) nach den Zielen von Analyse, ihm sei einerseits gelegen an »Vermittlung von Erkenntnis und Verständnis für Struktur, Qualität, Originalität, aber auch für Probleme des Werkes« (Motte 1996: 47). Analyse ist für ihn aber in zweiter Instanz auch eine »>Liebes-Erklärung‘; ich möchte zu Wort bringen, was und warum ich liebe« (ebd.). Diese Antworten von de la Motte weisen exemplarisch auf die beiden häufig in Analysen vorzufindenden impliziten Motive hin: Erstens will man Verständnis für das »Werk« erarbeiten, d.h. anderen ein ihm angemessenes, gar »richtiges« Verstehen seiner Geformtheit und somit seiner adäquaten Wahrnehmung mitteilen. Zweitens ist Analyse oft und in der Regel unhinterfragt eine Erforschung der eigenen Reaktion auf diese Musik. Dieser Zugang ist absolut legitim: »Aufklärung über sich selbst ist das Grundmotiv allen Fragens und damit aller Wissenschaft «, haben Helmut Rösing und Peter Petersen (2000: 16) die Ängstlichen und/ oder Nachdenkenden beruhigt. 
Auch in der Analyse populärer Musik sind beide Motive zu entdecken. So will Martin Pfleiderer (2009: 186) am Beispiel des Dub Reggae zeigen, dass ein »musikästhetischer Analyseansatz«, der ihm zufolge jenseits von normativen Ansprüchen stehen soll, die "große Attraktivität « dieser Musik begreifbar machen und anderen Hörern vermitteln könne. Angesichts der Begeisterung für den Gegenstand der Untersuchung ist jedoch Vorsicht angebracht, denn nicht selten führt sie beim kritischen Leser zum Eindruck einer pädagogisch verbrämten Missionierung. Im Nu rückt die Analyse populärer Musik somit in die Nähe der geradezu klassischen Analysesituation westlicher Kunstmusik, wo der Musikwissenschaftler »seine « Musik und ihre - für ihn! - speziellen Reize ${ }^{13}$ durch analytische Untersuchungen begründet, um sie dem Leser als »gute« Musik nahelegen zu können. Wenn darüber hinaus die Fachgrenzen überschritten werden, impliziert die asymmetrische Struktur dieser Kommunikation zwischen dem Experten einer- und dem Laien andererseits zudem - wie eh und je - eine wertvolle, weil von Diskursleitern ${ }^{14}$ wertvoll gesprochene Musik. Selbstverständlich soll dies nicht bedeuten, dass von nun an keine Analysen mehr über Lieblingsmusiken geschrieben werden sollten, von denen es nicht nur unter Popularmusikforschern einige geben dürfte. Ganz im Gegenteil! Gerade die Vertrautheit mit einer Musik kann eine immense Hilfe beim Erlangen von Erkenntnis sein, allerdings nur dann, wenn diese persönliche Verbundenheit erkannt und benannt wird.

Jede Analyse bedarf einer Fragestellung, die von einem Erkenntnisziel motiviert ist. So gewiss ein persönliches Interesse am Erkenntnisobjekt Beginn jeder Erkenntnissuche ist resp. sein muss, so darf doch dieser Anfang einer Vertiefung in den gewählten Gegenstand nicht dazu führen, dass Liebhaberei oder gar Geschmäcklerisches die wissenschaftliche Verpflichtung auf eine Reflexion und Ursachenermittlung zum Erliegen bringt. Zusammenhänge herzustellen, die das Besondere vor dem Hintergrund des Allgemeinen kritisch und für alle ersichtlich beleuchten, ist ein explizites Ziel von Analyse. Sie darf bei der persönlichen »Liebes-Erklärung «, der Analyse der eigenen ästhetischen Erfahrung beginnen, sie sollte jedoch dann ihr Ziel in

13 Ob dies klangliche, handwerkliche, künstlerische oder gar institutionelle sind, sei für den Moment dahingestellt.

14 Es stellen sich natürlich Zweifel ein, ob der Musikwissenschaftler ein solcher Diskursleiter je für die populäre Musik gewesen ist. Die wohl vielen der auf diesem Gebiet tätigen Wissenschaftler ständig begegnenden Anfragen von Radiostationen, Zeitungen oder Zeitschriften, ob man sich als "Experte für populäre Musik« zu einem bestimmten Thema äußern möge, belegen zumindest, dass eine Außensicht auf den eigenen Status existiert, die die oben stehende Warnung als berechtigt erscheinen lässt. 
einer reflektierten und - ich füge hinzu: gesellschaftlich relevanten Fragestellung begründen. Nicht nur kann Analyse somit als Methode der Musikästhetik auf der klanglichen Ebene begründen, welche Musikerfahrung sich daraus generierte, ${ }^{15}$ sondern sie ist auch ein probates Werkzeug auch der Musiksoziologie.

Bereits 1958 begreift Adorno in einem frühen Entwurf zur Musiksoziologie Analyse als deren geeignete Methode. Für ihn (2003b: 18) ist in Musik Gesellschaft enthalten: »Jeder Klang allein schon sagt Wir. « ${ }^{16}$ Dieser kollektive Gehalt sei von der Musiksoziologie gesellschaftlich zu dechiffrieren (vgl. ebd.: 12). Wie geht sie dabei vor? Musiksoziologie sei nicht in verschiedene Gebiete zu unterteilen, da der gesellschaftliche (Musik-)Prozess auch keine verschiedenen Sphären kenne. Deshalb gebe es keine starren Methoden, sondern diese richteten sich nach dem Gegenstand aus und legitimierten sich danach, was sie »daran aufleuchten« (ebd.: 9) lassen. Ein Musiksoziologe müsse ein doppeltes Verhältnis zur Musik einnehmen, d.h. sie sowohl von innen als auch von außen untersuchen. Von innen bedeutet, mithilfe der Analyse den musikalischen Sinn »gesellschaftlich zum Sprechen zu bringen« (ebd.: 12). Adorno gesteht zu, dass dies nicht einfach zu erlernen oder darzustellen sei, aber alle gelungenen Versuche sich durch eine innere Kohärenz - in meiner Deutung: den roten Faden des Erkenntnisinteresses und die »Kraft, die einzelnen Momente aufzuhellen«, auszeichneten. Als Bedingung einer produktiven Musiksoziologie sieht er das Verstehen der Sprache der Musik (zur Erinnerung: ein kontextsensitives Wissen um die musikalische Praxis), was wesentlich von der »Verfeinerung und Reflexion der musikalisch-analytischen Methoden« abhänge (ebd.; Herv. A.D.). Um Musik zu »verstehen«, bedürfe es nämlich der Fähigkeit zur »Introspektion« (ebd.: 15), über welche nur geschulte Experten (d.h. praktisch als auch theoretisch Gebildete) mit einer exzeptionellen und weiter ausgebildeten Fähigkeit zur »Selbstbetrachtung « (ebd.) verfügten. Von außen heißt für Adorno, bloß positivistische Äußerungen über Musik nicht als gegeben zu

15 Übrigens muss dies nicht auf das eigene Musikerleben beschränkt bleiben, sondern auch die explizierte Musikerfahrung anderer kann mit analytischen Ergebnissen argumentativ ergründet werden. »Why does it kick butt?«, lautet die berühmte Frage, die jeder Musikwissenschaftler eigentlich dem Hörer populärer Musik beantworten können sollte: »The chords, melodic contours, and metric structures must be grasped analytically or else one has no way of addressing how in material terms the music manages to skick butt« (McClary/Walser 1990: 290).

16 Ähnlich sieht dies auch Robert Walser, wenn er Analyse als Aufgabe beschreibt, den »human complexities that are registered in sounds « (2003: 38) gerecht zu werden. "You only have the problem of connecting music and society if you've separated them in the first place« (ebd.: 27). 
werten, sondern sie auf ihre Grundlagen der Aussageproduktion hin zu befragen. Musiksoziologie muss deshalb »die Fähigkeit zum immanenten musikalischen Mitvollzug kombinieren mit der Souveränität, das Phänomen von außen her zu verfremden und damit gesellschaftlich durchsichtig zu machen« (ebd.: 19).

Es versteht sich, dass Adornos Materialbegriff und seine elitäre Haltung gegenüber von ihm sogenannter »leichter Musik« einer umweglosen Übertragung auf die Analyse populärer Musik im Wege stehen. Und selbstverständlich ist die Aufgabe der »gesellschaftlichen Dechiffrierung « der Musik äußerst vage formuliert. Doch lässt sich festhalten, dass auch Adorno Analyse als ein Werkzeug der Erkenntnis begreift, das gesellschaftlich präformiertes Sprechen über Musik durch Analyse konfrontieren, d.h. belegen oder ablehnen kann. Analyse kann derart, gestützt durch die praktisch wie theoretisch erworbene Fähigkeit zur Kontextualisierung von Musik und durch den selbstreflexiven Umgang mit Material und Werkzeug der Analyse, zu einer musiksoziologischen Fragestellung methodisch beitragen. Ziel einer solchen, von Robert Walser (2003: 21) als »cultural analysis « bezeichneten Unternehmung ist es, unter Bezugnahme auf die analysierten Klangstrukturen aufzuzeigen, wie Diskurse soziale Bedeutung von Klang evozieren (und vice versa) und somit spezifische Musikerfahrungen ermöglichen. ${ }^{17}$ Eine so verstandene Analyse hat ein klares Erkenntnisinteresse: als Mittel, nicht Ziel der Popularmusikforschung an der Beschreibung kultureller Praxen und deren potentieller Veränderung beizutragen. ${ }^{18}$

\section{Konsequenzen: Diskursivierung der Analyse und radikale Selbstreflexivität}

Ein objektivierter Musikbegriff, der Musik zu untersuchen meint, wo er doch nur einen Musikbegriff vertritt, sowie die nur unzureichend hinterfragte

17 Auch Moore sieht dies ähnlich, allerdings zielt sein Bemühen in erster Linie auf das Verstehen der Musiker bzw. ihres Ausdruckswillens, weniger auf die kulturellen oder sozialen Ursachen und Gebräuche der Musik: »What good analysis does, it seems to me, is to show why it is that explicable musical structures can support the interpretations that we wish to put upon them. [...] And this is the project which, it seems to me, has become key in the last decade: to demonstrate why it is that the particular sounds, sound-complexes, sound-structures that musicians use are appropriate to the expressive circumstances they find themselves in, and therefore enable meaningful interpretations to be made of their actions in bringing about just those sounds, sound-complexes and soundstructures« (Moore 2007: xviii).

18 Ähnliche Ansprüche formuliert Blaukopf (1984: 21) für die Musiksoziologie. 
Position des forschenden Subjekts hindern daran, Analyse als ein Mittel zu mehr als - immerhin - der Erklärung eines musikästhetischen Verstehens zu begreifen. Erst durch eine Diskursivierung der Analyse, die das Sprechen über Musikbegriffe in die Analyse der klanglichen Strukturen ein- und rückbezieht, kann die lange überholte Trennung inner- und außermusikalischer Analyse überwunden werden, die einer historisch bedingten Auffassung entspringt, die mit der Idee der musikalischen Autonomie gegen Ende des 18. Jahrhunderts sich zu entwickeln begann (vgl. Eggebrecht 1973: 49). Die radikale Selbstreflexivität des Forschersubjekts muss zudem die Einsicht vermitteln, dass es durch Motive, Ziele und Methoden das klangliche Objekt rahmt und diskursiviert. Dieser Prozess ist expliziter Bestandteil jeder Analyse und gehört daher auch in ihre schriftliche Fassung.

In einer der wenigen Arbeiten, die sich analytisch mit elektronischer Tanzmusik auseinandersetzen, gibt Mark J. Butler (2006) ein anschauliches Beispiel für diese Forderungen. Butler erkennt in den anglophonen Popular Music Studies einen »reflexive turn« (ebd.: 16f.), der sich durch folgende Aspekte des Umgangs mit der zu analysierenden Musik auszeichne. Analyse wird von ihm als soziale Praxis bezeichnet, innerhalb der sich der Analysierende in Beziehung zu der Musik und anderen Diskursteilnehmern setzt. Durch intensiven Austausch mit anderen Wissenschaftlern, aber auch Musikern, Hörern, Tänzern usw. weiß der Analysierende um die Stärken wie Limitierungen seiner Arbeit. Zudem ist er sich bewusst, dass er seine multiplen Rollen nicht verleugnen kann, sondern versuchen muss, sie produktiv und offen in die analytische Argumentation zu integrieren; Butler spricht vom »explicit positioning of the analyst within the analysis (ebd.: 16; Herv. i. Orig.). Auf diese Weise hat nicht nur - wie gewohnt - der Wissenschaftler das Sprachrecht in der Analyse, sondern Butler erscheint in seinen Ausführungen auch als Hörer, Amateur-Musiker oder Teilnehmer an musikalischen Veranstaltungen, d.h. er berichtet über Musikerlebnisse beim Tanzen, beim Beobachten der Tänzer oder beim Beobachten der Beobachter der Tänzer während öffentlicher Aufführungen elektronischer Tanzmusik.

Diese radikale Selbstreflexivität als Voraussetzung und Teil der Analyse verlangt also, sich seiner Subjektivität und Rollendiversität, seiner Ziele der Analyse sowie der durch die Methode und Begriffe verursachten Effekte bewusst zu werden und sie zu benennen. Allgemein formuliert bedeutet es, die spezifische Situation der Analyse und der eigenen Rezeption dieser Musik hinsichtlich der subjektiven, sozialen, politischen, kulturellen oder ökonomischen Kontexte zu erhellen. Es wäre vermessen, diese Anforderungen zu konkretisieren; wer wie Butler glaubt, dass die Offenbarung sexueller Orientierung die eigene Analyse nachdrücklich verständlicher macht, kann, 
nein: soll das gerne tun. Wesentlicher als die inhaltliche ist nämlich die ethische Dimension dieser Handlungsmaxime, denn diese ausdrückliche (Selbst-)Positionierung des Forschenden versucht, den althergebrachten »god-trick of seeing everything from nowhere « (Haraway 1991: 189) ${ }^{19}$ einer objektivitätsfixierten Forschungslogik zu überwinden.

Die hier anklingende Idee radikaler Spezifität unterläuft somit einerseits das pauschalisierende Sprechen über Musik, sie muss allerdings andererseits anerkennen, dass sie nur einen Zugriff auf die untersuchte Musik darstellt. Es ist daher eine unvermeidliche Folge des Geforderten, sich die hier abzeichnende begrenzte Autorität des musikwissenschaftlichen Sprechens über populäre Musik - hier im Spezialfall der Analyse - angesichts der Vielzahl gültiger Interpretationen einzugestehen. Der sich bereits abzeichnende lautstarke Vorwurf, da »könne ja nun jeder kommen « und seine »Meinung « über populäre Musik zum Besten geben, offenbart einerseits das vom Zerfall bedrohte Anspruchsdenken einer Disziplin, die gewohnt war, ihre Deutungen der untersuchten Musik als allein gültige anzusehen. ${ }^{20}$ Andererseits können die Nörgler beruhigt werden: Analyse darf im Vergleich zum alltäglichen Sprechen eine besondere Gültigkeit beanspruchen, da sie ein wissenschaftliches Unternehmen ist und bleibt - insofern sie trotz des subjektiven und spezifischen Zugriffs, der in seiner Offenlegung die intersubjektive Nachvollziehbarkeit und somit eine handhabbare Objektivität anstrebt, weiterhin Ansprüche von Diskursivität, logischer Konsistenz und Überprüfbarkeit erfüllt.

Eigentümlicher- wie bezeichnenderweise finden wir diese Forderungen nach Reflexivität immer wieder in der Literatur zur - nicht nur populärmusikalischen - Analyse. Gruber fordert bspw. in der MGG:

»Die vom Musikanalytiker vorgenommene Analyse gibt nicht nur seine musikästhetische Position preis, sondern auch seinen historischen Standort, der mit der kompositionstechnischen Entwicklung nur bedingt standhält. [...] Eine Analyse ist nie abgeschlossen, ihr integrativer Bestandteil ist der Erfahrungshorizont des Analysierenden. Sie ist aber vollständig, wenn dieser Bezug erkannt, kritisch reflektiert und Teil der Analyse ist« (Gruber 1994: 579).

19 In der Übersetzung von Helga Kelle: »göttlichen Trick, alles von nirgendwo aus sehen zu können« (Haraway 1995: 81).

20 Die Auseinandersetzungen über die »richtige«, d.h. »beste« Analyse innerhalb der Musikwissenschaft vermochten nicht, die Expertenrolle der Disziplin im gesellschaftlichen Diskurs über Musik zu beeinträchtigten. Die Hinwendung zur zeitgenössischen Musik mit ihrer Betonung der klanglichen Ebene - und nicht zuletzt der teils vehemente Widerspruch der Komponisten gegen die vorgelegten Analysen - hat die Musikwissenschaft vor ähnliche Probleme gestellt, wie sie auch in der Analyse populärer Musik zu beobachten sind. 
Man kommt sich vor wie Don Quichotte im Kampf gegen die Windmühlen angesichts der vehementen Bezeugungen der Wissenschaft zur Verpflichtung auf die kritische Selbstreflexion und ihrer gleichzeitigen Zeugnisse der Folgenlosigkeit dieser Gelöbnisse. Die Feststellung, dass »kaum noch Anlass für ein distanziertes und zögerliches Verhältnis der Popmusikforschung zur musikalischen Analyse besteht « (Pfleiderer 2008: 169), mag einer deutschsprachigen Popularmusikforschung zustehen, die eine ähnliche Debatte über Analyse wie im anglophonen Raum bereits geführt hat. Derzeit aber muss diese Haltung sowohl als Symptom wie auch als Grund für die nach wie vor sich behauptende Pseudo-Reflektivität der Disziplin gedeutet werden.

Letztlich bleibt mir also nicht mehr, aber auch nicht weniger, als die lange bekannten Forderungen um die wenigen Ergänzungen (etwa die Diskurskompetenz) erweitert zur Umsetzung anzumahnen, gerade angesichts der zunächst einmal begrüßenswerten Zunahme von Analysen populärer Musik. Vielleicht ist gerade, weil Analyse sich momentan »under construction « befindet - um den Titel von Rappes (2010) Analyse des gleichnamigen Songs von Missy Elliott zu bemühen -, die Gelegenheit für meinen Eingriff günstig, den Analysierenden als Konstrukteur wahr- und ernstzunehmen. Wenn sich zudem die - oft unbewusst eingeschlichene - Idee des objektiv bestehenden, wert- wie gehaltvollen Werks, des Songs oder Tracks überwinden lässt hin zu einer Sicht auf die speziellen Prozesse der Bedeutungszuweisungen an Klang, kann es gelingen, Analyse als ein Instrument zu (be-)nutzen, das nicht das Verstehen von der Musik im Sinn hat, sondern hilft, das Verstehen des - vielfältigen und doch diskursiv geformten - Musikverstehens voranzutreiben, wie es als Aufgabe systematischer Musikwissenschaft formuliert worden (Motte-Haber 1982: 12) und für die Popularmusikforschung zu übernehmen ist.

\section{Literatur}

Adorno, Theodor W. (2003a). »Fragment über Musik und Sprache.«In: Ders. Musikalische Schriften I-III. Klangfiguren. Quasi una fantasia. Musikalische Schriften III. Hg. v. Rolf Tiedemann (= Gesammelte Schriften 16). Frankfurt/M.: Suhrkamp, S. 251-256.

Adorno, Theodor W. (2003b). »Ideen zur Musiksoziologie.«In: Ders. Musikalische Schriften I-III. Klangfiguren. Quasi una fantasia. Musikalische Schriften III. Hg. v. Rolf Tiedemann (= Gesammelte Schriften 16). Frankfurt/M.: Suhrkamp, S. 9-23.

Bent, lan D. / Pople, Anthony (2001). »Analysis. "In: The New Grove Dictionary of Music and Musicians Bd. 1. Hg. v. Stanley Sadie. London: Macmillan (2. Aufl.), S. 526-589. 
Blaukopf, Kurt (1984). Musik im Wandel der Gesellschaft. Grundzüge der Musiksoziologie. München u. Kassel: dtv u. Bärenreiter.

Brackett, David (1995). Interpreting Popular Music. Cambridge: Cambridge University Press.

Bryson, Bethany (1996). »>Anything but Heavy Metal.< Symbolic Exclusion and Musical Dislikes. «In: American Sociological Review 61, S. 884-899.

Butler, Mark J. (2006). Unlocking the Groove. Rhythm, Meter, and Musical Design in Electronic Dance Music. Bloomington / Indianapolis: Indiana University Press.

Cook, Nicholas (2001). »Analysing Performance and Performing Analysis." In: Rethinking Music. Hg. v. Nicholas Cook und Mark Everist. Oxford u. New York: Oxford University Press [Korr. Reprint].

Dahlhaus, Carl (1973). „Das >Verstehen< von Musik und die Sprache der Analyse.« In: Musik und Verstehen. Aufsätze zur semiotischen Theorie, Ästhetik und Soziologie der musikalischen Rezeption. Hg. v. Peter Faltin und Hans-Peter Reinecke. Köln: Arno Volk, S. 37-47.

DeNora, Tia (2000). Music in Everyday Life. Cambridge u.a.: Cambridge University Press.

Doehring, André (2006). „Schnittmuster der Präsentation populärer Musik. Überlegungen zur Analyse aktueller Popmusik am Beispiel Maxïmo Parks.«In: Cut and paste. Schnittmuster populärer Musik der Gegenwart. Hg. v. Dietrich Helms und Thomas Phleps (= Beiträge zur Popularmusikforschung 34). Bielefeld: Transcript, S. 115-134.

Doehring, André (2011). Musikkommunikatoren. Berufsrollen, Organisationsstrukturen und Handlungsspielräume im Popmusikjournalismus (= texte zur populären musik 7). Bielefeld: Transcript.

Eggebrecht, Hans Heinrich (1973). „Über begriffliches und begriffsloses Verstehen von Musik. «In: Musik und Verstehen. Aufsätze zur semiotischen Theorie, Ästhetik und Soziologie der musikalischen Rezeption. Hg. v. Peter Faltin und HansPeter Reinecke. Köln: Arno Volk, S. 48-57.

Eggebrecht, Hans Heinrich (2004). »Verstehen durch Analyse.«In: Musik und Verstehen. Hg. v. Christoph von Blumröder und Wolfram Steinbeck (= Spektrum der Musik 8). Laaber: Laaber, S. 18-27.

Elflein, Dietmar (2010). Schwermetallanalysen. Die musikalische Sprache des Heavy Metal ( = texte zur populären musik 6). Bielefeld: Transcript.

Erpf, Hermann (1951). »Analyse."In: Die Musik in Geschichte und Gegenwart. Bd. 1. Hg. v. Friedrich Blume. Kassel u. Basel: Bärenreiter, Sp. 449-454.

Everett, Walter (Hg.) (2000). Expression in Pop-Rock Music. A Collection of Critical and Analytical Essays. New York: Garland.

Frith, Simon (Hg.) (2004). Popular Music. Critical Concepts in Media and Cultural Studies. Vol. III. Popular Music Analysis. London u. New York: Routledge.

Geuen, Heinz / Hiemke, Sven (2001). „Von wechselnden Ansichten. Musikalische Semantik und künstlerische Konzeptualität im Song $A$ day in the life von John Lennon und Paul McCartney.«In: Pop \& Mythos. Pop-Kultur, Pop-Ästhetik, PopMusik. Hg. v. Heinz Geuen und Michael Rappe. Schliengen: Edition Argus, S. 175196.

Gruber, Gerold W. (1994). "Analyse. «In: Die Musik in Geschichte und Gegenwart. Sachteil Bd. 1. Hg. v. Ludwig Finscher. Kassel u.a. / Stuttgart, Weimar: Bärenreiter / Metzler (2. Aufl.), Sp. 577-591.

Haraway, Donna Jeanne (1991). "Situated Knowledges. The Science Question in Feminism and the Privilege of Partial Perspective. «In: Dies., Simians, Cyborgs, and Women. The Reinvention of Nature. New York: Routledge, S. 183-201 
[deutsch (1995). »Situiertes Wissen. Die Wissenschaftsfrage im Feminismus und das Privileg einer partialen Perspektive. «In: Die Neuerfindung der Natur. Primaten, Cyborgs und Frauen. Hg. u. eingeleitet von Carmen Hammer und Immanuel Stieß. Frankfurt/M. u. New York: Campus, S. 73-97].

Helms, Dietrich (2002). »Musikwissenschaftliche Analyse populärer Musik?« In: Musikwissenschaft und populäre Musik. Versuch einer Bestandsaufnahme. Hg. v. Helmut Rösing, Albrecht Schneider und Martin Pfleiderer (= Hamburger Jahrbuch für Musikwissenschaft 19). Frankfurt/M. u.a.: Lang, S. 91-103.

Horn, Wolfgang (1996). "Satzlehre, Musiktheorie, Analyse. Variationen über ein ostinates Thema.« In: Zum Problem und zu Methoden von Musikanalyse. Hg. v. Nico Schüler. Hamburg: von Bockel, S. 11-31.

Jost, Ekkehard (1986). »Über einige Probleme der Popularmusikforschung.«In: Ist Pop die Volksmusik von heute? Hg. v. Helmut Rösing (= Beiträge zur Popularmusikforschung 1). Hamburg, S. 30-37.

Kubik, Gerhard (1973). "Verstehen in afrikanischen Musikkulturen. «In: Musik und Verstehen. Aufsätze zur semiotischen Theorie, Ästhetik und Soziologie der musikalischen Rezeption. Hg. v. Peter Faltin und Hans-Peter Reinecke. Köln: Arno Volk, S. 171-188.

Kühn, Clemens (1993). Analyse lernen (= Studienbücher Musik 4). Kassel u.a.: Bärenreiter.

Manuel, Peter (2001). »II. World Popular Music. «In: The New Grove Dictionary of Music and Musicians. Bd. 20. Hg. v. Stanley Sadie. London: Macmillan (2. Aufl.), S. 153-166.

McClary, Susan / Walser, Robert (1990). "Start Making Sense! Musicology Wrestles with Rock. «In: On Record. Rock, Pop, and the Written Word. Hg. v. Simon Frith und Andrew Goodwin. London, New York: Routledge, S. 277-292.

Middleton, Richard (1990). Studying Popular Music. Milton Keynes: Open University Press.

Middleton, Richard (2001). »I. Popular Music in the West. "In: The New Grove Dictionary of Music and Musicians. Bd. 20. Hg. v. Stanley Sadie. London: Macmillan (2. Aufl.), S. 128-153.

Moore, Allan F. (2003a). »Introduction.«In: Analyzing Popular Music. Hg. v. dems. Cambridge u.a.: Cambridge University Press, S. 1-15.

Moore, Allan F. (Hg.) (2003b). Analyzing Popular Music. Cambridge u.a.: Cambridge University Press.

Moore, Allan F. (2007). »Introduction. «In: Critical Essays in Popular Musicology. Hg. v. dems. Aldershot: Ashgate, S. ix-xxii.

Moore, Allan F. (2009). »Interpretion: So what? In: The Ashgate Research Companion to Popular Musicology. Hg. v. Derek B. Scott. Farnham u. Burlington: Ashgate, S. 411-425.

Moraitis, Andreas (1994). Zur Theorie der musikalische Analyse. Frankfurt/M. u.a.: Peter Lang.

Motte, Diether de la (1996). »Zu den fünf Fragen.«In: Zum Problem und zu Methoden von Musikanalyse. Hg. v. Nico Schüler. Hamburg: von Bockel, S. 47-50.

Motte-Haber, Helga de la (1982). »Umfang, Methode und Ziel der Systematischen Musikwissenschaft. «In: Systematische Musikwissenschaft. Hg. v. Carl Dahlhaus und Helga de la Motte-Haber ( = Neues Handbuch der Musikwissenschaft 10). Wiesbaden und Laaber: Akademische Verlagsanstalt Athenaion und Laaber, S. 1-24.

Pfleiderer, Martin (2008). »Musikanalyse in der Popmusikforschung. Ziele, Ansätze, Methoden.«In: PopMusicology. Perspektiven der Popmusikwissenschaft. Hg. v. 
Christian Bielefeldt, Udo Dahmen und Rolf Grossmann. Bielefeld: Transcript, S. 153-171.

Rappe, Michael (2010). Under construction. Kontextbezogene Analyse afroamerikanischer Popmusik. 2 Bde. (= Musicolonia 6). Köln: Dohr.

Rauhe, Hermann (1974). Popularität in der Musik. Interdisziplinäre Aspekte musikalischer Kommunikation (= Musik und Gesellschaft 14). Karlsruhe: Braun.

Rienäcker, Gerd (1996). »Nachdenken über den Sinn musikalischer Analyse.« In: Zum Problem und zu Methoden von Musikanalyse. Hg. v. Nico Schüler. Hamburg: von Bockel, S. 39-45.

Rösing, Helmut / Petersen, Peter (2000). Orientierung Musikwissenschaft. Was sie kann, was sie will. Reinbek bei Hamburg: Rowohlt.

Schüler, Nico (Hg.) (1996). Zum Problem und zu Methoden von Musikanalyse. Hamburg: von Bockel.

Small, Christopher (1998). Musicking. The Meanings of Performing and Listening. Middletown: Wesleyan University Press.

Walser, Robert (2003). »Popular Music Analysis: Ten Apothegms and Four Instances. «In: Analyzing Popular Music. Hg. v. Allan F. Moore. Cambridge u.a.: Cambridge University Press, S. 16-38.

Warner, Timothy (2009). »Approaches to Analyzing Recordings of Popular Music. « In: The Ashgate Research Companion to Popular Musicology. Hg. v. Derek B. Scott. Farnham u. Burlington: Ashgate, S. 131-145.

Wicke, Peter (1997). »Populäre Musik.«In: Die Musik in Geschichte und Gegenwart. Sachteil Bd. 7. Hg. v. Ludwig Finscher. Kassel u.a. / Stuttgart, Weimar: Bärenreiter / Metzler (2. Aufl.), Sp. 1694-1704.

Wicke, Peter (2002). »Popmusik in der Theorie. Aspekte einer problematischen Beziehung. «In: Musikwissenschaft und populäre Musik. Versuch einer Bestandsaufnahme. Hg. v. Helmut Rösing, Albrecht Schneider und Martin Pfleiderer (= Hamburger Jahrbuch für Musikwissenschaft 19). Frankfurt/M. et al.: Lang, S. 61-73.

Wicke, Peter (2003). »Popmusik in der Analyse.« In: Acta Musicologica 75, H. 1, S. 107-126.

Wicke, Peter (2004). "Soundtracks. Popmusik und Pop-Diskurs. In: Was ist Pop? Zehn Versuche. Hg. v. Walter Grasskamp, Michaela Krützen und Stephan Schmitt. Frankfurt/M.: Fischer, S. 115-139.

\begin{abstract}
After a period of moderate usage, analysis recently seems to enjoy new demand as an appropriate tool for the study of popular music. Yet this paper claims first that still too little attention is being paid to the contexts and discourses of popular music which shape our notion of the music we want to analyse. Second, the analyst her/himself rarely questions her/his motives and goals of performing an analysis. In taking both claims seriously, analysis could be used for setting clear the aesthetic impressions popular music has to offer as well as advance again as a tool for sociomusicology, just like Adorno once proposed.
\end{abstract}

